\title{
Adversidades na Infância: Associação a Fatores Protetivos e Sintomas
}

\section{Internalizantes na Adultez}

\author{
Jaqueline Portella Giordani* \\ Universidade Federal do Rio Grande do Sul - UFRGS, Porto Alegre, RS, Brasil \\ ORCID: https://orcid.org/0000-0002-0231-2812 \\ Carolina Palmeiro Lima** \\ Universidade Federal do Rio Grande do Sul - UFRGS, Porto Alegre, RS, Brasil \\ ORCID: https://orcid.org/0000-0001-5103-2203 \\ Clarissa Marceli Trentini**** \\ Universidade Federal do Rio Grande do Sul - UFRGS, Porto Alegre, RS, Brasil \\ ORCID: https://orcid.org/0000-0002-2607-7132
}

\section{RESUMO}

Este estudo teve por objetivo discutir relações entre adversidades na infância e sintomas de depressão, ansiedade e estresse em uma amostra de adultos brasileiros, além dos fatores proximais e distais que poderiam explicar o caminho dessa relação da infância até a idade adulta. 510 participantes com idades entre 18 e 59 anos $(M D=30,64 ; D P=10,47)$ responderam a uma ficha de dados sociodemográficos, à Maltreatment and Abuse Exposure Scale, à DASS21 e à Social Readjustment Rating Scale, por coleta online e presencial. Os resultados indicaram que ter vivenciado adversidades na infância estava associado a maiores índices dos sintomas e à ideação suicida e que a violência psicológica e emocional se mostrou mais prevalente e com maior efeito em longo prazo. Percepção de rede de apoio social e psicoterapia são fatores que podem atuar de forma positiva e eventos estressores recentes podem intensificar o prejuízo sobre a saúde mental. São discutidas as implicações na identificação e intervenção sobre as adversidades na infância e no investimento nos fatores protetivos em longo prazo.

Palavras-chave: adversidades na infância, saúde mental, fatores protetivos.

\section{Childhood Adversities: Association with Protective Factors and}

\section{Internalizing Symptoms in Adulthood}

\begin{abstract}
This study aimed to discuss the relationships between childhood adversity and symptoms of depression, anxiety and stress in a sample of Brazilian adults, as well as the proximal and distal factors that could explain the path of this relationship from childhood to adulthood. 510 participants aged $18-59$ years $(\mathrm{M}=30.64 ; \mathrm{SD}=10.47)$ responded to a sociodemographic data
\end{abstract}


sheet, the Maltreatment and Abuse Exposure Scale, the DASS-21 and the Social Readjustment Rating Scale. The results indicated that experiencing childhood adversities is associated with higher indexes of symptoms and suicidal ideation, and that psychological and emotional violence was more prevalent and had a greater long-term effect. Perceptions of social support network and psychotherapy are factors that can act positively and recent stressful events can intensify the damage on mental health. Implications for identifying and intervening on childhood adversities and investing in long-term protective factors are discussed.

Keywords: childhood adversities, mental health, protective factors.

\title{
Adversidades en la Infancia: Asociación con Factores Protectores y
}

\author{
Síntomas Internalizantes en la Adultez
}

\section{RESUMEN}

Este estudio tuvo por objetivo discutir relaciones entre adversidades en la infancia y síntomas de depresión, ansiedad y estrés en una muestra de adultos brasileños, además de los factores proximales y distales que podrían explicar el camino de esa relación de la infancia hasta la edad adulta. 510 participantes con edades entre 18 y 59 años $(M=30,64, D E=10,47)$ respondieron a una ficha de datos sociodemográficos, a la Maltreatment and Abuse Exposure Scale, a la DASS-21 y a la Social Readjustment Rating Scale, por colecta online y presencial. Los resultados indicaron que haber vivido adversidades en la infancia estaba asociado a mayores índices de los síntomas y a la ideación suicida, y que la violencia psicológica y emocional se mostró más prevalente y con mayor efecto a largo plazo. La percepción de la red de apoyo social y la psicoterapia son factores que pueden actuar de forma positiva y los acontecimientos de estrés recientes pueden intensificar el perjuicio sobre la salud mental. Se discuten las implicaciones en la identificación e intervención sobre las adversidades en la infancia y en la inversión en los factores protectores a largo plazo.

Palabras clave: adversidades en la infancia, salud mental, factores protectores.

As adversidades na infância podem ser descritas como eventos que ocorrem ou na família ou no contexto social da criança que provocam sofrimento e prejuízo na saúde física ou psicológica, podendo variar em intensidade e tendem a ser crônicos (Kalmakis \& Chandler, 2014). Sabe-se que são amplamente prevalentes e frequentemente coocorrem em populações em geral, independentemente de contexto cultural ou nível socioeconômico (Kessler et al., 2010, Finkelhor, Turner, Shattuck, \& Hamby, 2015). No Brasil, em 2015, o número de denúncias de violações de direitos ao Disque 100 (Módulo Criança e Adolescente) 
foi de 153 mil. Destas, 72,8\% foram sobre situações de negligência, 45,7\% de violência psicológica, 42,4\% de violência física e 21,3\% de violência sexual (ABRINQ, 2017).

Algumas formas de abuso são consideradas formas aceitáveis de prática disciplinar parental, ou seja, há alto índice de subnotificação e esses números podem ser ainda maiores. Segundo o Fundo das Nações Unidas para a Infância [UNICEF] (2017), em todo o mundo, por volta de 1,1 bilhão de cuidadores (cerca de 1 a cada 4) admitem acreditar na necessidade do castigo físico como forma de disciplina. Até o momento apenas 60 países adotaram uma legislação que proíbe totalmente o uso de castigos corporais em casa. Essa falta de proibições legais é um sinal claro de que a disciplina violenta, seja física ou verbal, continua sendo uma forma amplamente desconhecida e banalizada de violência contra crianças, sendo sua ocorrência comumente não comunicada aos órgãos competentes (Finkelhor, Turner, Shattuck, \& Hamby, 2015; UNICEF, 2017).

A infância é considerada um período sensível no decurso da vida, durante o qual uma adversidade significativa pode lançar cadeias de desvantagens nas relações ao longo do ciclo vital que, então, têm efeitos cumulativos sobre a saúde, incluindo a saúde mental, ao longo do tempo (Umberson, Williams, Thomas, Liu, \& Thomeer, 2014). Como hipótese explicativa, alguns estudos sugerem um aumento da reatividade emocional e fisiológica aos estressores após quaisquer tipos de maus-tratos na infância (Bandoli et al., 2017; Heleniak, Jenness, Vander Stoep, McCauley, \& McLaughlin, 2016). Entretanto, a resposta ao estresse parece não depender exclusivamente das vivências precoces adversas, como também de acontecimentos estressores recentes, além de ser possível que essas vivências estressoras - na infância e depois na idade adulta - estejam associadas (Bandoli et al., 2017).

Estudos epidemiológicos têm encontrado associações significativas entre adversidades na infância e piores condições de saúde, especialmente saúde mental, em adultos (Bowes, Joinson, Wolke, \& Lewis, 2015; Kessler et al., 2010; Nanni, Uher, \& Danese, 2012). Os achados de estudos populacionais são de que indivíduos que experimentaram adversidades na infância estão em risco elevado de desenvolver algum transtorno mental internalizante ao longo da vida em comparação com indivíduos sem essa exposição, e as chances de desenvolver essa sintomatologia são maiores à medida em que a exposição à adversidade aumenta (Green et al., 2010; Kessler et al., 2010; McLaughlin, Conron, Koenen, \& Gilman, 2010; McLaughlin, 2016). É bem descrito esse efeito em longo prazo em relação a sintomas internalizantes, mesmo considerando-se o controle de outros fatores, em casos de depressão (Coles, Lee, Taft, Mazza, \& Loxton, 2015; Lindert et al., 2014; McLafferty, O’Neill, Murphy, Armour, \& Bunting, 2018; Nanni et al., 2012; Norman et al., 2012) e de ansiedade (Benedetti 
et al., 2014; Simon et al., 2009; Spinhoven et al., 2010), além de estar associado à ideação suicida (Côelho et al., 2016; McLafferty et al., 2018; Salokangas, Luutone, Heinimaa, From, \& Hietala, 2019; Stansfeld et al., 2017). Autores ressaltam a importância da compreensão das adversidades na infância tanto de forma específica, como a sua coocorrência, a fim de se entender fatores que possam contribuir para o desfecho na saúde mental geral e qualidade de vida em longo prazo (Kessler et al., 2010; Merrick et al., 2017). Ainda, para intervenção e prevenção nos casos de vivências adversas na infância, são necessárias a identificação e a descrição de como atuam os mecanismos de proteção na relação adversidadedesenvolvimento (Banyard, Hamby, \& Grych, 2017).

Como fatores que possam diminuir o risco de prejuízo que pode ser provocado na saúde mental nos quadros citados, no caso de vivências adversas precoces, tem-se compreendido que uma rede social diversificada e de apoio pode reduzir o risco de sintomas de depressão, sugerindo uma possível via de proteção (Platt, Keyes, \& Koenen, 2014). A literatura tem indicado que essa presença de boas relações interpessoais ao longo da vida (Collishaw et al., 2007; Davies, Consortium, Fletcher, Goodyer, \& Jones, 2017) pode diminuir o impacto das adversidades. Ademais, ter tido acesso à psicoterapia tem sido associado a uma menor probabilidade de prejuízo na saúde mental em adultos que vivenciaram adversidades (Raposo, Mackenzie, Henriksen, \& Afifi, 2014). A adversidade na infância parece carregar uma capacidade única de prejudicar o bem-estar psicológico adulto, tanto independentemente quanto de forma cumulativa, incluindo desvantagem social e experiências adultas estressantes (Nurius, Green, Logan-Greene, \& Borja, 2015). Considerando os fatores expostos, o objetivo desse estudo foi o de investigar a associação de diferentes tipologias de adversidades na infância em uma amostra de adultos brasileiros a sintomas internalizantes na vida adulta, considerando fatores proximais como estressores recentes, rede de apoio social e acesso à psicoterapia.

\section{Método}

Este estudo caracterizou-se por ser um levantamento quantitativo, transversal e correlacional, com coleta realizada tanto de forma online, via survey, quanto de forma presencial, por conveniência. 


\section{Participantes}

Participaram do estudo 510 sujeitos, com idades entre 18 e 59 anos $(M D=30,64 ; D P$ $=10,47$ ), de ambos os sexos (sendo 77,3\% do sexo feminino). Entre os participantes, 57,5\% eram pessoas solteiras e 21,6\% eram casadas. Em relação à formação educacional, mais de um terço da amostra estava cursando ou havia completado pós-graduação.

\section{Instrumentos}

Ficha de Dados Sociodemográficos. Utilizada para coletar informações não contempladas nas demais escalas utilizadas, comportamentos de risco na adolescência, fatores de risco e proteção na infância e adolescência, abordando aspectos biosociodemográficos dos participantes (como sexo, idade, escolaridade), assim como aqueles relacionados à educação, saúde física e mental, trabalho, rede de apoio social, psicoterapia, ideação suicida.

\section{Maltreatment and Abuse Exposure Scale (MAES) (Kluwe-Schiavon, Viola, \&} Grassi-Oliveira, 2016). A escala MAES é constituída de 52 itens que avaliam o grau de exposição a dez tipos de abuso: negligência emocional, abuso emocional não-verbal, maustratos físicos parentais, abuso verbal dos pais, abuso emocional dos pares, bullying físico de pares, negligência física, abuso sexual, testemunho de violência interparental e testemunho de violência contra irmãos. A escala original exibiu propriedades psicométricas confiáveis, sendo que os escores de correlação variaram de bom a excelente na confiabilidade teste-reteste do instrumento para o grau geral de exposição a diferentes tipos de maus-tratos (Teicher \& Parigger, 2015). Para essa amostra o instrumento exibiu bom índice de confiabilidade e consistência interna, com alfa de Cronbach de 0,90.

Depression, Anxiety and Stress Scale-21 (DASS-21) (Lovibond \& Lovibond, 2004). $\mathrm{O}$ instrumento possui três escalas com sete itens cada, totalizando 21 itens que avaliam sintomas de sofrimento mental em adultos, especificamente: ansiedade, depressão e estresse na semana anterior à pesquisa. A versão brasileira da DASS-21 foi traduzida e adaptada por Vignola e Tucci (2014). A escala apresentou boa consistência interna para essa amostra, com alfas de Cronbach de 0,92 para subescala de depressão, 0,88 para a de estresse, 0,86 para a de ansiedade e 0,94 para a escala total.

\section{Social Readjustment Rating Scale (Holmes \& Rahe, 1967) - tradução de Lipp}

(1984). Instrumento de avaliação de eventos vitais recentes que indica a frequência de ocorrência de eventos estressores no ano anterior à pesquisa e atribui uma pontuação arbitrária 
diferenciada a cada evento, considerando seu potencial estressor. Para essa amostra o instrumento exibiu fidedignidade adequada, com alfa de Cronbach com o valor de 0,75.

\section{Procedimentos}

Os aspectos éticos que garantem a integridade dos participantes foram assegurados de acordo com legislações brasileiras, tendo como base a Resolução n.510/2016 do Conselho Nacional de Saúde (Brasil, 2016). O estudo foi aprovado pelo Comitê de Ética em Pesquisa do Instituto de Psicologia da UFRGS, sob número 85159718.7.0000.5334 do CAAE e com parecer número 2.609.341. Os participantes foram convidados a participar do estudo, sendo esclarecida a voluntariedade da participação, a garantia de sigilo das informações pessoais e a possibilidade de desistência a qualquer momento. A aplicação presencial de parte dos questionários se deu de forma coletiva em turmas de Educação de Jovens e Adultos da rede pública de educação de Porto Alegre, e o restante por questionário online, via survey - com duração de aproximadamente 60 minutos. Foi disponibilizada assistência por parte da equipe de pesquisa, no caso de algum participante necessitar de apoio durante a realização da coleta de dados. No caso da coleta online, o participante poderia interromper o preenchimento a qualquer tempo, e solicitar encaminhamentos em caso de desconforto com alguma questão. Foi solicitado aos participantes o Consentimento Livre e Esclarecido.

\section{Análise de Dados}

Estatísticas descritivas e inferenciais foram realizadas no programa SPSS, segundo objetivos do estudo. Os dados foram submetidos à análise estatística descritiva. Buscou-se conhecer o perfil pessoal, econômico, social, de saúde e de vivências das adversidades na infância dos participantes. Os dados foram analisados inicialmente segundo a ocorrência prévia ou não de adversidades na infância. Foi realizada uma estratificação por grupos, na qual o primeiro grupo referiu-se a pessoas que vivenciaram entre nenhuma e quatro adversidades (G1 -Exposição baixa/moderada a adversidades, $n=367$ ), e o segundo foi composto por pessoas que relataram cinco ou mais adversidades compreendidas na escala MAES (G2 -Exposição alta/múltiplas adversidades, $n=143$ ). Foram realizadas análises inferenciais de diferenças entre os grupos através de testes de qui-quadrado (para avaliação da associação entre variáveis categóricas) e teste t de Student (para avaliação de diferenças de médias entre os dois grupos). Para a realização de uma Regressão Linear Múltipla, foi 
utilizado o escore na escala de sintomas internalizantes, sendo que quanto maior o escore maior a sintomatologia apresentada, como variável dependente. As variáveis independentes na análise foram: idade, sexo, renda, escolaridade, estressores recentes, religiosidade, acesso à psicoterapia, rede de apoio social e adversidades na infância. Foi utilizado o método stepwise em três passos - variáveis sociodemográficas, fatores da vida adulta e por fim adversidades na infância e rede de apoio na infância e adolescência.

\section{Resultados}

Das dez adversidades pesquisadas, os participantes relataram ter experienciado, em média, 2,97 tipologias de vivências adversas $(D P=2,64)$ compreendidas na escala MAES. Do total da amostra, $1 \%$ relatou ter sofrido os dez tipos de adversidades investigadas. As mais relatadas foram negligência emocional $(42,2 \%)$ e abuso emocional por pares $(42,4 \%)$. Negligência física foi a menos relatada $(8,8 \%)$. Relativamente a tratamentos para saúde mental, $68,8 \%$ dos participantes referiu já ter realizado psicoterapia, sendo que $12 \%$ o fizeram durante a infância, 21,2\% durante a adolescência e 56,7\% após os dezoito anos de idade. A etapa em que foi realizado o tratamento não provocou diferença nos desfechos nas análises.

Os resultados indicaram diferenças entre os grupos segundo o grau de exposição a adversidades em relação a variáveis na adultez. Foram analisadas as diferenças em relação ao relato de ideação suicida - em qualquer ponto da vida. Um teste de qui-quadrado indicou que, no grupo com exposição alta a adversidades (G2), 80,4\% dos sujeitos já pensou em se matar, contra $48,8 \%$ de pessoas que pensaram nisso no grupo com baixa/moderada exposição a adversidades $(\mathrm{G} 1), \operatorname{com} \chi^{2}(1, N=510)=42,209, p<0,001, \phi=0,288$. A razão de chances de o sujeito ter apresentado ideação suicida em algum ponto da vida foi 4,3 vezes maior se a exposição a adversidades na infância foi alta, em comparação a pessoas com baixa/moderada exposição $(\mathrm{p}<0,001)$. Análises de teste $\mathrm{t}$ indicaram diferenças entre os grupos também para a sintomatologia de depressão (d), de estresse (e) e de ansiedade (a), sendo que as médias de G1 foram maiores $\mathrm{MD}(\mathrm{d})=5,77(\mathrm{DP}=5,69), \mathrm{MD}(\mathrm{e})=7,23(\mathrm{DP}=5,14), \mathrm{MD}(\mathrm{a})=3,64(\mathrm{DP}=$ 4,16) do que as de $\mathrm{G} 2 \mathrm{MD}(\mathrm{d})=9,14(\mathrm{DP}=6,07)), \mathrm{MD}(\mathrm{e})=10,85(\mathrm{DP}=5,13), \mathrm{MD}(\mathrm{a})=6,40$ $(\mathrm{DP}=5,09)$, sendo $\mathrm{t}(\mathrm{d})=-5,883, \mathrm{p}<0,001, d$ de Cohen $=0,572, \mathrm{t}(\mathrm{e})=-7,144, \mathrm{p}<0,001, d$ de Cohen $=0,704$, e $\mathrm{t}(\mathrm{a})=-5,772, \mathrm{p}<0,001, d$ de Cohen $=0,593$.

Além disso, com o objetivo de verificar quais variáveis proximais e distais estavam independentemente associadas à sintomatologia de depressão, ansiedade e estresse, foi realizada uma regressão linear múltipla, com os resultados expressos na Tabela 1. Esse 
modelo foi significativo $F(11,441)=21,08 ; \mathrm{p}<0,001$, e as variáveis incluídas no modelo explicaram 32,8\% ( $\mathrm{r}^{2}$ ajustado $\left.=0,328\right)$ da variação nos escores da escala DASS-21.

\begin{tabular}{|c|c|c|c|c|c|}
\hline \multirow{2}{*}{$\begin{array}{l}\text { Variáveis preditoras } \\
\text { incluidas na equação }\end{array}$} & \multicolumn{5}{|c|}{ DASS-21 } \\
\hline & $B$ & Beta & $t$ & $p$ & IC $95 \%$ \\
\hline Rede de apoio (atual) & $-10,403$ & $-0,188$ & $-4,653$ & $<0,001$ & $-14,797--6,009$ \\
\hline Abuso emocional pares & 0,665 & 0,169 & 3,731 & $<0,001$ & $0,315-1,015$ \\
\hline Abuso emocional não-verbal & 0,967 & 0,166 & 3,384 & 0,001 & $0,405-1,528$ \\
\hline Estressores recentes & 0,015 & 0,149 & 3,637 & $<0,001$ & $0,007-0,023$ \\
\hline Rede de apoio (adolescência) & $-4,607$ & $-0,136$ & $-3,020$ & 0,003 & $-7,604--1,609$ \\
\hline Idade & $-0,168$ & $-0,122$ & $-2,543$ & 0,011 & $-0,299--0,038$ \\
\hline Psicoterapia & $-3,432$ & $-0,112$ & $-2,708$ & 0,007 & $-5,923--0,941$ \\
\hline Sexo feminino & 3,701 & 0,109 & 2,644 & 0,008 & $0,950-6,452$ \\
\hline Escolaridade & $-0,549$ & $-0,056$ & $-1,129$ & 0,260 & $-1,504-0,406$ \\
\hline Renda & 0,000 & $-0,038$ & $-0,702$ & 0,483 & $-0,001-0,000$ \\
\hline Etnia (não-branco) & 0,504 & 0,015 & 0,381 & 0,703 & $-2,096-3,104$ \\
\hline Constante & 43,067 & - & 6,661 & $<0,001$ & $30,359-55,774$ \\
\hline
\end{tabular}

Nota. As variáveis estão dispostas conforme o valor de Beta, ou seja, a força de contribuição do item para o escore de sintomas internalizantes segundo a escala DASS-21.

\section{Discussão}

Primeiramente, é importante salientar que, apesar de muitos trabalhos na área dos maus-tratos e de violações de direitos na infância e adolescência, as definições conceituais e suas operacionalizações em termos de medida ainda são diversas - com a inclusão de diferentes adversidades conforme cada pesquisa (Kalmakis \& Chandler, 2014). Assim, o uso de escalas padronizadas, como a MAES, pode facilitar a compreensão dos dados de pesquisa e as diferenças entre países/amostras, por investigar de forma sistemática as diferentes tipologias de adversidades a que crianças e adolescentes podem estar sujeitos. Considerando isso, a prevalência de adversidades na infância encontrada neste estudo vai ao encontro dos achados de outras pesquisas. As adversidades ampliadas também podem ser consideradas para além dos maus-tratos parentais e, nesses casos, os índices podem ser superiores a $80 \%$ de 
exposição a adversidades na infância (Merrick et al., 2017), da mesma forma que os resultados deste estudo.

A polivitimização parece ser um fator que intensifica o potencial prejuízo sobre a saúde mental. Ela se refere à experiência de múltiplas vitimizações de diferentes tipos pelo mesmo sujeito, como violência sexual, abuso físico, intimidação, testemunho de violência familiar e exposição à violência comunitária, e não apenas a múltiplos episódios da mesma tipologia adversa (Turner, Shattuck, Finkelhor, \& Hamby, 2017). Os resultados do presente estudo demonstraram que os sujeitos que compuseram o grupo com alta exposição a adversidades na infância apresentaram índices maiores em todos os sintomas internalizantes analisados, além de chances aumentadas para ideação suicida em algum ponto da vida, em relação a sujeitos que não sofreram adversidades ou sofreram exposição baixa/moderada. Esse índice acumulado de adversidades na infância e na adolescência parece ser o ponto chave na análise do efeito em longo prazo dessas vivências (Kessler et al., 2010; Turner et al., 2017).

Já o modelo explicativo encontrado na análise de regressão, incluiu diversas variáveis que contribuem tanto negativa quanto positivamente com a alteração dos índices de sintomatologia internalizante. O modelo que melhor explicou esta relação incluiu menor percepção de rede de apoio na vida adulta, ter sofrido abuso emocional de pares e abuso emocional não-verbal parental na infância, maior índice de estressores recentes, menor percepção de rede de apoio social na adolescência, ser mais novo, não ter realizado psicoterapia, ser do sexo feminino, ter menor escolaridade, renda financeira média mais baixa e ser não-branco como variáveis independentemente associadas à maior sintomatologia de depressão, ansiedade e estresse. Ou seja, mesmo quando considerados diversos fatores proximais, as adversidades relacionadas a abuso psicológico ainda parecem provocar prejuízo sobre a saúde mental - mesmo anos depois do ocorrido. Esse modelo explicou 32,8\% da variação nos índices de sintomatologia de depressão, ansiedade e estresse. Isso indica que outros fatores certamente estão implicados na apresentação dessa sintomatologia, além dos analisados neste artigo.

Os achados no presente estudo contribuem com a vasta literatura internacional, inclusive de estudos epidemiológicos (Evans, Li, \& Whipple, 2013), que tem encontrado associações entre maus-tratos na infância e maiores índices de sintomas internalizantes na idade adulta, sugerindo que as consequências dessa vitimização na infância podem persistir por várias décadas após sua ocorrência (Chapman et al., 2004; Infurna et al., 2016; Sheikh, Abelsen, \& Olsen, 2016a; Sheikh, Abelsen, \& Olsen, 2016b). Neste estudo, mesmo quando considerados eventos estressores no último ano, as adversidades na infância permaneceram 
como variáveis explicativas na alteração do índice de sintomas internalizantes - inclusive, contribuindo para o modelo mais do que esses eventos proximais de vida. Outros estudos têm encontrado resultados similares, sendo que o efeito das adversidades na infância parece se manter mesmo ao serem considerados eventos recentes traumáticos (Bandoli et al., 2017). Já se compreende que as adversidades somadas a eventos recentes podem estar associadas à manifestação mais severa dos sintomas psicopatológicos (Asselmann et al., 2018).

Em relação aos fatores associados ao desfecho em saúde mental neste estudo, a rede de apoio social foi o fator com maior contribuição negativa à sintomatologia internalizante, indicando ser um fator protetivo ao desenvolvimento. Sabe-se que as relações sociais podem contribuir ou reduzir as disparidades sociais na saúde (Umberson \& Karas Montez, 2010), desde que os relacionamentos com familiares e amigos são particularmente importantes para a saúde mental devido à sua natureza íntima e contínua (Thoits, 2011). Aspectos negativos de rede social percebida têm sido associados a transtornos afetivos e de ansiedade (Ford, Clark, \& Stansfeld, 2011). A contribuição da rede de apoio social percebida no modelo encontrado nesta pesquisa é mais alta que outros fatores protetivos considerados nas análises. A explicação possível, segundo a literatura, é que vivência adversa na infância lançaria uma cascata de risco que aumentaria a probabilidade de exposição ao estresse na idade adulta (Umberson et al., 2014) e afetaria a percepção de suporte social (Patwardhan et al., 2017), o que, por sua vez, degradaria a qualidade dos relacionamentos na adultez (Umberson et al., 2014). Apesar de os resultados indicarem associação entre essas variáveis, sem um caminho de causalidade definido, mesmo estudos longitudinais têm encontrado resultados que mostram um efeito direto de abusos na infância na saúde mental e no bem-estar em adultos, mas que parte desse efeito pode sim ser mediado pela percepção de apoio social (Sheikh et al., 2016a; Sheikh et al., 2016b).

Outro fator protetivo considerado nas análises foi o acesso à psicoterapia, estando associado negativamente ao aumento do índice de sintomatologia internalizante independentemente do período de vida em que o tratamento foi realizado. Estudos acerca dos caminhos que ligam a vivência de adversidades ao tratamento psicoterápico encontraram evidências de que os abusos vivenciados na infância estão associados a pior funcionamento psicológico atual mesmo em grupos clínicos (Heinonen, Knekt, Härkänen, Virtala, \& Lindfors, 2018; Schilling, Weidner, Schellong, Joraschky \& Pöhlmann, 2015) e também ao resultado do tratamento na psicoterapia (Schilling et al., 2015). Ainda, parece ser importante que as adversidades precoces sejam consideradas durante o tratamento para o aumento da capacidade de alguém se beneficiar efetivamente de psicoterapia (Heinone et al., 2018). 
Ademais, a escolaridade apareceu como negativamente associada no modelo em relação à sintomatologia estudada, podendo ser considerada um dos fatores nessa amostra que se mostrou protetivo à saúde mental. Em outros estudos, o tempo de estudo formal também foi encontrado como um dos fatores associados a menor prejuízo na saúde mental (Cerqueira \& Coelho, 2015; Taillieu, Brownridge, Sareen, \& Afifi, 2016). Além disso, ser do sexo feminino apareceu no modelo como positivamente associado à sintomatologia internalizante. Essas diferenças entre homens e mulheres têm sido encontradas tanto em relação às vivências adversas (Alves, Dutra, \& Maia, 2013; Cerqueira et al., 2016; Waiselfisz, 2015) quanto aos efeitos na adultez (Alves et al., 2013; Benedetti et al., 2014; Currie \& Spatz Widom, 2010). Também nos resultados está a diferença entre raças/etnias, e é provável que as relações e a saúde dos adultos não-brancos sejam mais prejudicadas pelas adversidades na infância porque o estresse precoce é agravado pelo estresse adicional da exposição diária ao racismo e discriminação ao longo da vida (Schilling \& Christian, 2014; Williams \& Mohammed, 2009). Essas diferenciações são importantes para futuras análises e para atuação com populações específicas (Alves et al., 2013).

A associação, especialmente do abuso emocional ainda na infância, a um risco aumentado para desenvolvimento de transtornos internalizantes anos ou décadas depois da vitimização também tem sido identificada em outras pesquisas (Bandoli, 2017; Taillieu et al., 2016; Vachon, Krueger, Rogosh, \& Cichetti, 2015). Na amostra deste estudo, o abuso emocional não-verbal parental (como: ter sido castigado, trancado em algum lugar, ter responsabilidades de adultos, e pais difíceis de agradar ou sem tempo/interesse) foi a adversidade mais relatada, juntamente a abuso por pares. A literatura indica que o abuso emocional pode ser caracterizado por um padrão repetitivo no comportamento do cuidador da criança/adolescente, comunicando que ela não tem importância, suas imperfeições, que é malamada ou indesejada, que corre perigo e somente têm valor quando atende às necessidades do outro (Brodski \& Hutz, 2017; Taillieu, Brownridge, Sareen, \& Afifi, 2016). Da mesma forma que o indicado pelos resultados desse estudo, pesquisas populacionais longitudinais (Sheik et al., 2016; Taillieu et al., 2016) e estudos de meta-análises (Infurna et al., 2016) têm indicado que o abuso psicológico na infância pode ser relativamente mais importante para explicar a variação na saúde mental em comparação com outras formas de vitimização. Apesar da alta prevalência encontrada nos resultados desta pesquisa e seu efeito em longo prazo sobre a saúde mental, os abusos emocionais ainda são extremamente subnotificados, dentre as formas de maus tratos infantis. Dados do Ministério da Saúde (Waiselfisz, 2015) acerca dos registros de atendimento do Sistema Único de Saúde no campo das violências contra crianças e 
adolescentes dão conta de que a violência física absorve cerca de 35,4\% dos atendimentos. Violências psicológicas são notificadas em apenas 15,2\% dos registros.

Meta-análise de pesquisas das últimas duas décadas acerca da associação entre adversidades na infância e saúde mental em adultos encontrou que mais da metade dos casos globais de depressão e ansiedade são potencialmente atribuíveis a maus-tratos autodeclarados na infância, e que uma redução de 10 a 25\% desses abusos poderia auxiliar na prevenção de 31,4 a 80,3 milhões de casos de depressão e ansiedade em todo o mundo (Li, D’Arcy, \& Meng, 2015). Os achados do presente estudo foram coerentes com os de pesquisas populacionais e transculturais, que indicam que grande parte da população vivencia algum tipo de adversidade, que elas estão correlacionadas, e que o prejuízo pode se dar em longo prazo sobre a saúde mental, especificamente em relação a sintomas internalizantes (Kalmakis \& Chandler, 2014; Kessler et al., 2010, Finkelhor, Turner, Shattuck, \& Hamby, 2015).

Finalmente, algumas limitações acerca deste estudo devem ser destacadas. A confiabilidade nas informações fornecidas pelos participantes deve levar em consideração tanto a capacidade destes de relembrar as situações adversas vivenciadas na infância e adolescência, como o seu interesse em divulgar esses acontecimentos, mesmo com a garantia de sigilo das informações. Além disso, salienta-se que os resultados indicaram associação entre as variáveis discutidas, sem que seja possível indicar um fluxo de causalidade. Para estudos futuros, sugere-se a inserção de outras variáveis que possam estar relacionadas às adversidades na infância, bem como a avaliação também longitudinal dos fatores associados não apenas à vitimização, mas à perpetração dessas formas de violência, além de outros indicadores de saúde mental.

\section{Considerações Finais}

Este estudo investigou a prevalência de adversidades na infância em uma amostra de brasileiros e a associação dessas vivências com a saúde mental na adultez, considerando certos fatores indicados pela literatura como pertencentes ao caminho explicativo dessa relação. Essa associação, e as variáveis incluídas no modelo explicativo encontrado têm sido também identificadas em estudos em outras regiões do país e em pesquisas internacionais, indicando que essas relações não se devem a fatores apenas individuais e/ou regionais, mas que se constituem em fatores macrossistêmicos. Prevenir a violência contra as crianças requer uma grande mudança no que as sociedades consideram práticas aceitáveis de disciplina e nos relacionamentos interpessoais, sobretudo no que tange à comunicação violenta. Salienta-se a 
importância da não-banalização de nenhuma forma de violência contra crianças e adolescentes - especialmente em relação às violências psicológicas. Investir em relacionamentos sociais positivos parece ser um caminho possível para a diminuição do efeito das adversidades na infância sobre a saúde mental dos sujeitos vítimas, além do acompanhamento psicoterapêutico das vítimas de quaisquer formas de abuso ou de privação durante seu percurso de desenvolvimento, para que possa ser reduzido o impacto negativo que essa forma de violência provoca na qualidade de vida.

Embora a última década tenha visto uma melhoria acentuada na disponibilidade de dados sobre a violência contra crianças, certos tipos permanecem subpesquisados e subnotificados (UNICEF, 2017). Sendo assim, é urgente a investigação de adversidades de forma ampliada, para compreensão de como todos os contextos de desenvolvimento em que o sujeito está inserido podem ser determinantes no processo de adultez e na saúde mental ao longo do ciclo vital. Parece ser necessário que estratégias de prevenção e intervenção em nível populacional enfatizem o abuso emocional, que ocorre com alta frequência, mas é menos punível do que outros tipos de maus-tratos infantis (Vachon et al., 2015). Os resultados deste estudo e das pesquisas apresentadas reforçam a necessidade de iniciativas legais, de saúde e de políticas sociais para reduzir a prevalência de adversidades na infância e para a intervenção em casos de identificação dessas situações.

\section{Referências}

Alves, J., Dutra, A., \& Maia, Â. (2013). História de adversidade, saúde e psicopatologia em reclusos: Comparação entre homens e mulheres. Ciência \& Saúde Coletiva, 18(3), 701-709. doi:10.1590/S1413-81232013000300016

Asselmann, E., Stender, J., Grabe, H. J., König, J., Schmidt, C. O., Hamm, A. O., \& PanéFarré, C. A. (2018). Assessing the interplay of childhood adversities with more recent stressful life events and conditions in predicting panic pathology among adults from the general population. Journal of affective disorders, 225, 715-722. doi:10.1016/j.jad.2017.08.050

Associação Brasileira dos Fabricantes de Brinquedos [ABRINQ]. (2017). Cenário da Infância e Adolescência no Brasil 2017. Osasco, SP: Nywgraf. Recuperado de http://www.chegadetrabalhoinfantil.org.br/wp-content/uploads/2017/03/Cenario-2017PDF.pdf

Bandoli, G., Campbell-Sills, L., Kessler, R. C., Heeringa, S. G., Nock, M. K., Rosellini, A. J., 
... \& Stein, M. B. (2017). Childhood adversity, adult stress, and the risk of major depression or generalized anxiety disorder in US soldiers: A test of the stress sensitization hypothesis. Psychological medicine, 47(13), 2379-2392. doi:10.1017/S0033291717001064

Banyard, V., Hamby, S., \& Grych, J. (2017). Health effects of adverse childhood events: Identifying promising protective factors at the intersection of mental and physical well-being. Child Abuse \& Neglect, 65, 88-98. doi:10.1016/j.chiabu.2017.01.011

Benedetti, F., Poletti, S., Radaelli, D., Pozzi, E., Giacosa, C., \& Smeraldi, E. (2014). Adverse childhood experiences and gender influence treatment seeking behaviors in obsessive: Compulsive disorder. Comprehensive psychiatry, 55(2), 298-301. doi:10.1016/j.comppsych.2013.08.028

Bowes, L., Joinson, C., Wolke, D., \& Lewis, G. (2015). Authors' reply to Males. BMJ: British Medical Journal (Online), 351, h3648. doi:10.1136/bmj.h3648

Brasil. Ministério da Saúde. Conselho Nacional de Saúde. (2016, maio 24). Resolução nº 510, de 7 de abril de 2016. Diário Oficial União Seção 1, 98, 44-46. Recuperado de http://conselho.saude.gov.br/resolucoes/2016/Reso510.pdf\&gt

Brodski, S. K., \& Hutz, C. S. (2017). Novas perspectivas sobre o abuso emocional. Diaphora, 16(1), 13-19. Recuperado de http://www.sprgs.org.br/diaphora/ojs/index.php/diaphora/article/view/119/124

Cerqueira, D., Ferreira, H., Lima, R. S. D., Bueno, S., Hanashiro, O., Batista, F., \& Nicolato, P. (2016). Atlas da violência 2016. Rio de Janeiro: IPEA.

Cerqueira, D. R. C., \& Coelho, D. S. C. (2015). Redução da Idade de Imputabilidade Penal, Educação e Criminalidade. Rio de Janeiro: Ipea.

Chapman, D. P., Whitfield, C. L., Felitti, V. J., Dube, S. R., Edwards, V.J., \& Anda, R.F. (2004). Adverse childhood experiences and the risk of depressive disorders in adulthood. Journal of Affective Disorders, 82(2), 217-225. doi:10.1016/j.jad.2003.12.013

Coêlho, B. M., Andrade, L. H., Borges, G., Santana, G. L., Viana, M. C., \& Wang, Y. P. (2016). Do Childhood Adversities Predict Suicidality? Findings from the General Population of the Metropolitan Area of São Paulo, Brazil. PloS One, 11(5), 1-18. doi:10.1371/journal.pone.0155639

Coles, J., Lee, A., Taft, A., Mazza, D., \& Loxton, D. (2015). Childhood sexual abuse and its association with adult physical and mental health: Results from a national cohort of young Australian women. Journal of interpersonal violence, 30(11), 1929-1944. 
doi:10.1177/0886260514555270

Collishaw, S., Pickles, A., Messer, J., Rutter, M., Shearer, C., \& Maughan, B. (2007). Resilience to adult psychopathology following childhood maltreatment: Evidence from a community sample. Child abuse \& neglect, 31(3), 211-229. doi:10.1016/j.chiabu.2007.02.004

Currie, J., \& Spatz Widom, C. (2010). Long-term consequences of child abuse and neglect on adult economic well-being. Child maltreatment, 15(2), 111-120. doi:10.1177/1077559509355316

Davies, D., Consortium, N. S. P. N., Fletcher, P., Goodyer, I., \& Jones, P. (2017). SA7: Different Longitudinal Relationships Between Childhood Adversity, Adolescent/Adult Social Support, and Dimensions of Depressive and Psychotic Symptoms in 2 General Population Cohorts. Schizophrenia Bulletin, 43(suppl.1), S115-S115. doi:10.1093/schbul/sbx023.006

Evans, G. W., Li, D., \& Whipple, S. S. (2013). Cumulative risk and child development. Psychological Bulletin, 139(6), 1342-1396. doi:10.1037/a0031808

Finkelhor, D., Turner, H. A., Shattuck, A., \& Hamby, S. L. (2015). Prevalence of childhood exposure to violence, crime, and abuse: Results from the national survey of children's exposure to violence. JAMA Pediatrics, 169(8), 746-754. doi:10.1001/jamapediatrics.2015.0676

Ford, E., Clark, C., \& Stansfeld, S. A. (2011). The influence of childhood adversity on social relations and mental health at mid-life. Journal of affective disorders, 133(1-2), 320327. doi:10.1016/j.jad.2011.03.017

Green, J. G., McLaughlin, K. A., Berglund, P. A., Gruber, M. J., Sampson, N. A., Zaslavsky, A. M., \& Kessler, R. C. (2010). Childhood adversities and adult psychiatric disorders in the national comorbidity survey replication I: Associations with first onset of DSMIV disorders. Archives of General Psychiatry, 67(2), 113-123. doi:10.1001/archgenpsychiatry.2009.186

Heinonen, E., Knekt, P., Härkänen, T., Virtala, E., \& Lindfors, O. (2018). Associations of early childhood adversities with mental disorders, psychological functioning, and suitability for psychotherapy in adulthood. Psychiatry research, 264, 366-373. doi: 10.1016/j.psychres.2018.04.011

Heleniak, C., Jenness, J. L., Vander Stoep, A., McCauley, E., \& McLaughlin, K. A. (2016). Childhood maltreatment exposure and disruptions in emotion regulation: A transdiagnostic pathway to adolescent internalizing and externalizing 
Jaqueline Portella Giordani, Carolina Palmeiro Lima, Clarissa Marceli Trentini

psychopathology. Cognitive therapy and research, 40(3), 394-415. doi:10.1007/s10608-015-9735-Z

Holmes, T. H., \& Rahe, R. H. (1967). The social readjustment rating scale. Journal of psychosomatic research, 11(2), 213-218. doi:10.1016/0022-3999(67)90010-4

Infurna, M. R., Reichl, C., Parzer, P., Schimmenti, A., Bifulco, A., \& Kaess, M. (2016). Associations between depression and specific childhood experiences of abuse and neglect: a meta-analysis. Journal of affective disorders, 190, 47-55. doi:10.1016/j.jad.2015.09.006

Kalmakis, K. A., \& Chandler, G. E. (2014). Adverse childhood experiences: Towards a clear conceptual meaning. Journal of Advanced Nursing, 70(7), 1489-1501. doi:10.1111/jan.12329

Kessler, R. C., McLaughlin, K. A., Green, J. G., Gruber, M. J., Sampson, N. A., Zaslavsky, A. M., ... Williams, D. R. (2010). Childhood adversities and adult psychopathology in the WHO world mental health surveys. British Journal of Psychiatry, 197(5), 378385. doi:10.1192/bjp.bp.110.080499

Kluwe-Schiavon, B., Viola, T. W., \& Grassi-Oliveira, R. (2016). Cross-cultural adaptation of the Maltreatment and Abuse Chronology of Exposure (MACE) scale to Brazilian Portuguese. Trends in psychiatry and psychotherapy, 38(1), 33-39. doi:10.1590/22376089-2015-0051

Li, M., D'arcy, C., \& Meng, X. (2016). Maltreatment in childhood substantially increases the risk of adult depression and anxiety in prospective cohort studies: Systematic review, meta-analysis, and proportional attributable fractions. Psychological medicine, 46(4), 717-730. doi:10.1017/S0033291715002743

Lipp, M. E. N. (1984). Stress e suas implicações. Estudos em Psicologia (Campinas), 1, 5-19. Recuperado de https://www.researchgate.net/publication/313768075_Stress_e_suas_implicacoes

Lindert, J., von Ehrenstein, O. S., Grashow, R., Gal, G., Braehler, E., \& Weisskopf, M. G. (2014). Sexual and physical abuse in childhood is associated with depression and anxiety over the life course: Systematic review and meta-analysis. International journal of public health, 59(2), 359-372. doi:10.1007/s00038-013-0519-5

Lovibond, S. H., \& Lovibond, P. F. (2004). Manual for the Depression Anxiety Stress Scales (4th ed.). Sydney: Psychology Foundation.

McLafferty, M., O’Neill, S., Murphy, S., Armour, C., \& Bunting, B. (2018). Population attributable fractions of psychopathology and suicidal behaviour associated with 
childhood adversities in Northern Ireland. Child abuse \& neglect, 77, 35-45. doi:10.1016/j.chiabu.2017.12.015

McLaughlin, K. A. (2016). Future directions in childhood adversity and youth psychopathology. Journal of Clinical Child \& Adolescent Psychology, 45(3), 361-382. doi:10.1080/15374416.2015.1110823

McLaughlin, K. A., Conron, K. J., Koenen, K. C., \& Gilman, S. E. (2010). Childhood adversity, adult stressful life events, and risk of past-year psychiatric disorder: A test of the stress sensitization hypothesis in a population-based sample of adults. Psychological Medicine, 40(10), 1647-1658. doi:10.1017/S0033291709992121

Merrick, M. T., Ports, K. A., Ford, D. C., Afifi, T. O., Gershoff, E. T., \& Grogan-Kaylor, A. (2017). Unpacking the impact of adverse childhood experiences on adult mental health. Child abuse \& neglect, 69, 10-19. doi:10.1016/j.chiabu.2017.03.016

Nanni, V., Uher, R., \& Danese, A. (2012). Childhood maltreatment predicts unfavorable course of illness and treatment outcome in depression: A meta-analysis. American Journal of Psychiatry, 169(2), 141-151. doi:10.1176/appi.ajp.2011.11020335

Norman, R. E., Byambaa, M., De, R., Butchart, A., Scott, J., \& Vos, T. (2012). The long-term health consequences of child physical abuse, emotional abuse, and neglect: A systematic review and meta-analysis. PLoS medicine, 9(11), 1-31. doi:10.1371/journal.pmed.1001349

Nurius, P. S., Green, S., Logan-Greene, P., \& Borja, S. (2015). Life course pathways of adverse childhood experiences toward adult psychological well-being: A stress process analysis. Child Abuse Neglect, 45, 143-153. doi:10.1016/j.chiabu.2015.03.008

Patwardhan, I., Mason, W. A., Savolainen, J., Chmelka, M. B., Miettunen, J., \& Järvelin, M. R. (2017). Childhood cumulative contextual risk and depression diagnosis among young adults: The mediating roles of adolescent alcohol use and perceived social support. Journal of adolescence, 60, 16-26. doi:10.1016/j.adolescence.2017.07.008

Platt, J., Keyes, K. M., \& Koenen, K. C. (2014). Size of the social network versus quality of social support: Which is more protective against PTSD?. Social psychiatry and psychiatric epidemiology, 49(8), 1279-1286. doi:10.1007/s00127-013-0798-4

Raposo, S. M., Mackenzie, C. S., Henriksen, C. A., \& Afifi, T. O. (2014). Time does not heal all wounds: Older adults who experienced childhood adversities have higher odds of mood, anxiety, and personality disorders. The American Journal of Geriatric Psychiatry, 22(11), 1241-1250. doi:10.1016/j.jagp.2013.04.009

Salokangas, R. K. R., Luutonen, S., Heinimaa, M., From, T., \& Hietala, J. (2019). A study on 
the association of psychiatric diagnoses and childhood adversities with suicide risk. Nordic Journal of Psychiatry, 73(2), 125-131. doi:10.1080/08039488.2018.1493748

Schilling, S., \& Christian, C. W. (2014). Child physical abuse and neglect. Child and Adolescent Psychiatric Clinics of North America, 23(2), 309-319. doi:10.1016/j.chc.2014.01.001

Schilling, C., Weidner, K., Schellong, J., Joraschky, P., \& Pöhlmann, K. (2015). Patterns of childhood abuse and neglect as predictors of treatment outcome in inpatient psychotherapy: A typological approach. Psychopathology, 48(2), 91-100. doi:10.1159/000368121

Sheikh, M. A., Abelsen, B., \& Olsen, J. A. (2016a). Clarifying associations between childhood adversity, social support, behavioral factors, and mental health, health, and well-being in adulthood: A population-based study. Frontiers in psychology, 7, 1-24. doi:10.3389/fpsyg.2016.00727

Sheikh, M. A., Abelsen, B., \& Olsen, J. A. (2016b). Differential recall bias, intermediate confounding, and mediation analysis in life course epidemiology: An analytic framework with empirical example. Frontiers in psychology, 7, 1-16. doi:10.3389/fpsyg.2016.01828

Simon, N. M., Herlands, N. N., Marks, E. H., Mancini, C., Letamendi, A., Li, Z., .. \& Stein, M. B. (2009). Childhood maltreatment linked to greater symptom severity and poorer quality of life and function in social anxiety disorder. Depression and anxiety, 26(11), 1027-1032. doi:10.1002/da.20604

Spinhoven, P., Elzinga, B. M., Hovens, J. G., Roelofs, K., Zitman, F. G., van Oppen, P., \& Penninx, B. W. (2010). The specificity of childhood adversities and negative life events across the life span to anxiety and depressive disorders. Journal of affective disorders, 126(1-2), 103-112. doi:10.1016/j.jad.2010.02.132

Stansfeld, S., Clark, C., Smuk, M., Power, C., Davidson, T., \& Rodgers, B. (2017). Childhood adversity and midlife suicidal ideation. Psychological Medicine, 47(2), 327-340. doi:10.1017/S0033291716002336

Taillieu, T. L., Brownridge, D. A., Sareen, J., \& Afifi, T. O. (2016). Childhood emotional maltreatment and mental disorders: Results from a nationally representative adult sample from the United States. Child Abuse \& Neglect, 59, 1-12. doi:10.1016/j.chiabu.2016.07.005

Teicher, M. H., \& Parigger, A. (2015). The 'Maltreatment and Abuse Chronology of Exposure' (MACE) Scale for the retrospective assessment of abuse and neglect during 
Jaqueline Portella Giordani, Carolina Palmeiro Lima, Clarissa Marceli Trentini

development. PLoS one, 10(2), 1-37. doi:10.1371/journal.pone.0117423

Thoits, P. A. (2011). Mechanisms linking social ties and support to physical and mental health. Journal of health and social behavior, 52(2), 145-161. doi:10.1177/0022146510395592

Turner, H. A., Shattuck, A., Finkelhor, D., \& Hamby, S. (2017). Effects of poly-victimization on adolescent social support, self-concept, and psychological distress. Journal of interpersonal violence, 32(5), 755-780. doi:10.1177/0886260515586376

Umberson, D., \& Karas Montez, J. (2010). Social Relationships and Health: A Flashpoint for Health Policy. Journal of Health and Social Behavior, 51(suppl.1), S54-S66. doi:10.1177/0022146510383501

Umberson, D., Williams, K., Thomas, P. A., Liu, H., \& Thomeer, M. B. (2014). Race, gender, and chains of disadvantage: Childhood adversity, social relationships, and health. Journal of Health and Social Behavior, 55(1), 20-38. doi:10.1177/0022146514521426

United Nations Children's Fund. (2017). A familiar face: Violence in the lives of children and $\begin{array}{llll}\text { adolescents. New } & \text { York: Unicef. Recuperado de }\end{array}$ https://www.Unicef.org/publications/files/Violence_in_the_lives_of_children_and_ad olescents.pdf

Vachon, D. D., Krueger, R. F., Rogosch, F. A., \& Cicchetti, D. (2015). Assessment of the harmful psychiatric and behavioral effects of different forms of child maltreatment. JAMA psychiatry, 72(11), 1135-1142. doi:10.1001/jamapsychiatry.2015.1792

Viola, T. W., Salum, G. A., Kluwe-Schiavon, B., Sanvicente-Vieira, B., Levandowski, M. L., \& Grassi-Oliveira, R. (2016). The influence of geographical and economic factors in estimates of childhood abuse and neglect using the childhood trauma questionnaire: a worldwide meta-regression analysis. Child Abuse \& Neglect, 51, 1-11. doi:10.1016/j.chiabu.2015.11.019

Vignola, R. C. B., \& Tucci, A. M. (2014). Adaptation and validation of the depression, anxiety and stress scale (DASS) to Brazilian Portuguese. Journal of affective disorders, 155, 104-109. doi:10.1016/j.jad.2013.10.031

Waiselfisz, J. J. (2012). Mapa da violência: Crianças e adolescentes do Brasil. Rio de Janeiro:

Flacso.

Recuperado

de https://www.mapadaviolencia.org.br/pdf2012/MapaViolencia2012_Criancas_e_Adole scentes.pdf

Waiselfisz, J. J. (2015). Violência letal contra as crianças e adolescentes do Brasil. Rio de Janeiro: Flacso. Recuperado de 
Jaqueline Portella Giordani, Carolina Palmeiro Lima, Clarissa Marceli Trentini

http://www.mapadaviolencia.org.br/publicacoes/Violencia_Letal_web.pdf

Williams, D. R., \& Mohammed, S. A. (2009). Discrimination and racial disparities in health: Evidence and needed research. Journal of behavioral medicine, 32(1), 20-47. doi:10.1007/s10865-008-9185-0

\section{Endereço para correspondência}

\section{Jaqueline Portella Giordani}

Rua Ramiro Barcelos, 2600, sala 225, $2^{\circ}$ andar, Santa Cecília, Porto Alegre - RS, Brasil. CEP 90035-003

Endereço eletrônico: jaquelinegiordani@gmail.com

\section{Carolina Palmeiro Lima}

Rua Ramiro Barcelos, 2600, sala 225, 2º andar, Santa Cecília, Porto Alegre - RS, Brasil. CEP 90035-003

Endereço eletrônico: psi.carolinalima@gmail.com

\section{Clarissa Marceli Trentini}

Rua Ramiro Barcelos, 2600, sala 225, 2º andar, Santa Cecília, Porto Alegre - RS, Brasil. CEP 90035-003

Endereço eletrônico: clarissatrentini@ terra.com.br

Recebido em: 03/09/2019

Reformulado em: 02/06/2020

Aceito em: 10/06/2020

\section{Notas}

* Doutora em PPG em Psicologia/UFRGS. Psicóloga no Colégio de Aplicação da UFRGS e tutora de núcleo da Psicologia na RIS Saúde Mental Coletiva/UFRGS.

** Possui graduação em Psicologia Pela UNISINOS (2016) e mestrado pelo PPG em Psicologia da UFRGS (2019). Doutoranda em Psicologia (UFRGS).

*** Professora Associada e Diretora do Instituto de Psicologia da UFRGS. Coordenadora do Núcleo de Estudos em Avaliação Psicológica e Psicopatologia.

Este artigo de revista Estudos e Pesquisas em Psicologia é licenciado sob uma Licença Creative Commons Atribuição-Não Comercial 3.0 Não Adaptada. 